\title{
ANÁLISIS DE VARIABLES DE FLUJO VEHICULAR EN PUNTOS CRÍTICOS DE ACCIDENTALIDAD
}

\section{ANALYSIS OF VEHICLE FLOW VARIABLES AT CRITICAL ACCIDENT POINTS}

\author{
MSc. Jhan Rojas Suárez*, MSc. Sofia Orjuela Abril **, \\ MSc. Gaudy Prada Botia* \\ * Universidad Francisco de Paula Santander, Facultad de Ingenierías. \\ Av. Gran Colombia 12E-96 Colsag, San José de Cúcuta, Norte de Santander, Colombia. \\ 0375 776655. E-mail: \{jhanpierorojas, gaudycarolinapb\}@ufps.edu.co \\ ** Universidad Francisco de Paula Santander, Facultad de Ciencias Empresariales, \\ Av. Gran Colombia 12E-96 Colsag, San José de Cúcuta, Norte de Santander, Colombia. \\ 0375 776655. E-mail: sofiaorjuela@ufps.edu.co
}

\begin{abstract}
Resumen: En esta investigación se analiza el comportamiento del flujo vehicular en un corredor vial, determinado los índices de accidentalidad, morbilidad, mortabilidad, velocidad vehicular y el volumen de vehículos que transitan por este sector, estableciendo los puntos críticos de accidentalidad vial mediante el análisis estadístico basado en los informes suministrados, gráficas y comparaciones para un mayor entendimiento de la situación que corren riesgo los conductores y peatones, mientras que se visualiza que cada año aumenta un $1 \%$ la tasa de muertes por accidentes de tráfico, la velocidad vehicular se encuentra en el $72 \mathrm{~km} / \mathrm{h}$ y el volumen vehicular fue descendiendo.
\end{abstract}

Palabras clave: Accidentalidad, flujo vehicular, morbilidad y mortalidad.

\begin{abstract}
This research analyzes the behavior of vehicle flow in a road corridor, determining accident rates, morbidity, vehicle speed and the volume of vehicles that pass through this sector, establishing the critical points of road accidents through statistical analysis based on the reports provided, graphs and comparisons for a better understanding of the situation that drivers and pedestrians are at risk, while it is visualized that each year increases by $1 \%$ the rate of deaths from traffic accidents, the vehicle speed is at $72 \mathrm{~km} / \mathrm{h}$ and the vehicle volume was declining.
\end{abstract}

Keywords: Accident, morbidity, mortality and vehicle flow

\section{INTRODUCCIÓN}

Las grandes intersecciones son causadas principalmente por factores como la topografía y los esquemas de planificación de carreteras, facilitando los problemas de tráfico, lo que resultaría en una menor eficiencia del tráfico en las intersecciones, así como en el confusión y conflicto entre los diferentes medios de transporte, y la seguridad de cruzar la frontera (Liu et al., 2018) . El nivel potencial de seguridad vial en determinados tipos de intersecciones puede depender de: normas de tráfico claramente especificadas, número de puntos de colisión potenciales, velocidades permitidas, visibilidad para los usuarios de los flujos de tráfico en las 
intersecciones, ángulos de intersección de los flujos de tráfico que colisionan, método de utilización de determinados canales de tráfico, tipo de subordinación del tráfico, etc (Szczuraszek y Klusek, 2019); ocasionando los accidentes de tráfico más peligrosos y con mayor tasa de mortalidad. La Organización Mundial de la Salud (OMS) ha clasificado los accidentes de tráfico como la novena causa más común de muerte en todo el mundo (Roslan et al., 2018).

Los jóvenes son más vulnerables a los accidentes de tráfico debido a: la inexperiencia, la toma de isletas, el exceso de velocidad, las distracciones, las drogas y los alcoholes, entre otros (Dano, 2018). El exceso de velocidad es la principal causa de víctimas, es decir, el 66,5 \% de todos los accidentes de tráfico, y el $61 \%$ de las muertes se deben al exceso de velocidad (Farhan et al., 2018), aunque, una situación inesperada de accidente también puede ocurrir por los accidentes de vuelco, que tienen la especificidad de que el vehículo ya no puede ser controlado, su movimiento se realiza deslizándose y rodando la carrocería (Gaiginschi et $a l ., 2017)$. Las estadísticas muestran que alrededor del $40 \%$ de los las muertes de los ocupantes de los coches se producen en el impacto frontal (Suman et al., 2017). (11. Bermeo, WL, Jr, AB de Souza, Fernández T, Honorio D, Nogueira dos Reis L, Barreto L. 2017).

En este estudio, se analizará las variables de flujo vehicular que indicen en accidente de tráfico, además, de indicadores de accidentabilidad y mortabilidad que va en aumento cada año; debido a que los choferes no tienen en cuenta los límites de velocidad y curvas peligrosas por una indebida infraestructura; de esta forma se concluirá el riesgo que corre el peatonal y los choferes en estas vías.

\section{MATERIALES Y MÉTODOS}

El presente proyecto tiene como objetivo analizar el comportamiento del flujo vehicular en el corredor vial, determinando los índices de accidentalidad, morbilidad, mortalidad para la población y el parque vehicular, índice de accidentalidad por kilometraje, accidentalidad en intersecciones y la severidad de tomar intersecciones, velocidad y volumen del tránsito del año 1 y 2; para una población compuesta por un número variable de ciudadanos y medios de transporte que diariamente transitan estas vías, luego de realizar la verificación y análisis de la señalización existente, condiciones riesgosas que no se ajusten a las normas de seguridad, formatos de conteo y toma de velocidades, carteras topográficas y encuestas de satisfacción de las vías aplicadas a ciudadanos que usualmente las transitan (Pacheco y Mendoza, 2018).

\section{RESULTADOS Y DISCUSIÓN}

\subsection{Análisis del índice de accidentes de tránsito}

El municipio de Los Patios cuenta con una población de 76524 habitantes; según la secretaria de tránsito y transporte se encuentran registrados 65900 vehículos. Durante el año 1 se presentaron 103 accidentes con 95 heridos y 8 muertos, en el año 2 se presentaron 75 accidentes con 72 heridos y 13 muertos. Con los datos anteriores se determina los índices con respecto a la población, índices con respecto al parque vehicular (Tabla 2), índices de accidentalidad con respecto al kilometraje de viaje IA/K (Tabla 3) y comparación de los índices (Figura 3).

\subsection{1 Índices con respecto a la población}

P1= Población

$\mathrm{Ia}=$ Índice de accidentalidad

Imorb = Índice de morbilidad

Imort = Índice de mortalidad

$\frac{1 Q}{\mathrm{P} 1}=\frac{\# \text { Accidentes en el año*10000 }}{\# \text { habitantes }}$

$\frac{\text { Imorb }}{\mathrm{P} 1}=\frac{\# \text { Heridos en el año*10000 }}{\# \text { habitantes }}$

$\frac{\text { Imort }}{\mathrm{P} 1}=\frac{\text { \#Muertes en el año*10000 }}{\text { \# habitantes }}$

Tabla 1. Índices con respecto a la población

\begin{tabular}{cccc}
\hline Año & Accidentalidad & Morbilidad & Mortalidad \\
\hline 1 & 134,59 & 124,14 & 10,45 \\
\hline 2 & 98,00 & 81,02 & 16,96 \\
\hline
\end{tabular}




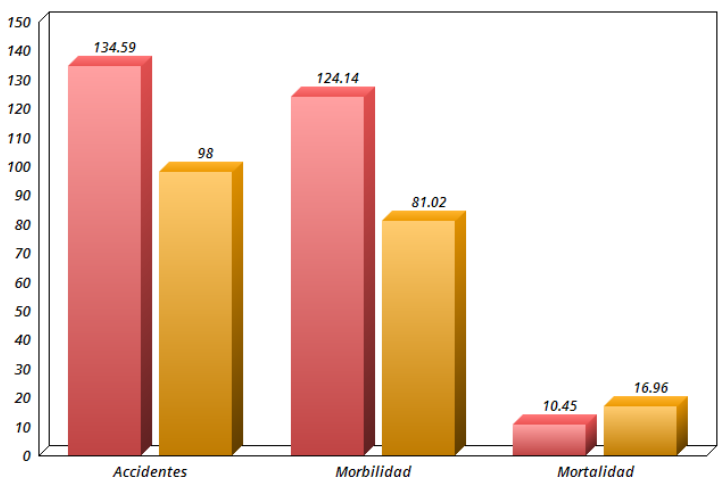

$\square$ Año $1 \square$ Año 2

Fig 1. Índices con respecto a la población. Fuente: Elaboración propia.

A medida del tiempo, se reducen los accidentes de tráfico y morbilidad, pero, incrementa el riesgo de morir en un accidente de tráfico cada año, aumentando la deficiencia y la seguridad vial de la población, según lo representado en la Tabla 1 y Figura 1.

\subsection{2 Índices con respecto al parque vehicular}

P2 = Parque vehicular

$\frac{1 Q}{\mathrm{~V}}=\frac{\text { \#Accidentes en el año*10000 }}{\# \text { vehículos registrados }}$

$\frac{\text { Imorb }}{\mathrm{V}}=\frac{\# \text { Heridos en el año } * 10000}{\# \text { vehículos registrados }}$

$\frac{\text { Imort }}{\mathrm{V}}=\frac{\# \text { Muertes en el año*10000 }}{\# \text { vehículos registrados }}$

$\underline{\text { Tabla 2. Índices con respecto al parque vehicular }}$

\begin{tabular}{cccc}
\hline Año & Accidentalidad & Morbilidad & Mortalidad \\
\hline 1 & 15,63 & 14,42 & 1,21 \\
\hline 2 & 11,38 & 9,41 & 1,97 \\
\hline
\end{tabular}

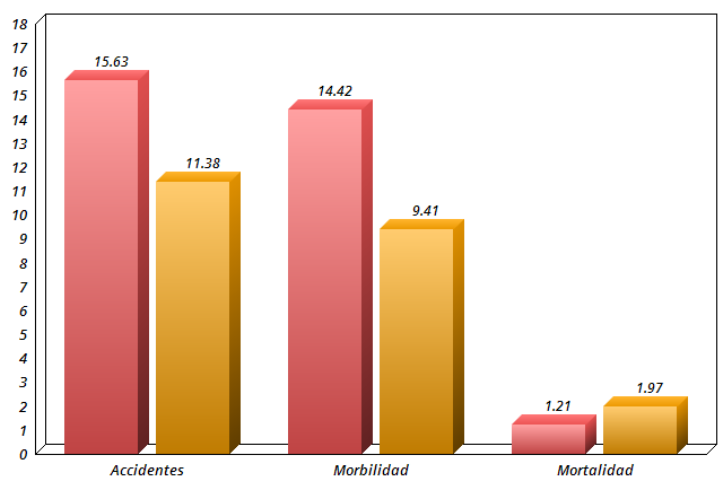

$\square$ Año $1 \square$ Año 2

Fig 2. Indexes with respect to vehicle fleet. Fuente: Elaboración propia.

En la Tabla 2 y Figura 2, es evidente que los accidentes con respecto a la flota de vehículos disminuyeron en comparación con los 2 años, aun así, la mortalidad de los mismos está aumentando, haciendo más peligroso tener un accidente cada vez más años.

3.1.3 Índices de accidentalidad con respecto al kilometraje de viaje.

$\mathrm{K}=$ Kilometraje

$\mathrm{VK}=$ kilometraje de viaje

VK = Velocidad y densidad promedio anual

TPD = Tránsito Promedio Diario

$\mathrm{L}=$ Kilometraje promedio

$\frac{I Q}{K}=\frac{\text { \#Muertos en el aîo*10000 }}{\text { \#VK }}$

$\mathrm{Vk}=\mathrm{TPD}(365)(\mathrm{L})$

Tabla 3. Indices de accidentalidad IA/K

\begin{tabular}{cc}
\hline Año & IA/K \\
\hline 1 & 1,08 \\
\hline 2 & 1,55 \\
\hline
\end{tabular}

3.1.4 Índices de accidentalidad con respecto al número de vehículos que entran a una intersección

VEI $=$ Vehículos entrando en una intersección

$\mathrm{V}=$ Promedio de vehículos al año

$\frac{1 Q}{V E I}=\frac{\# \text { Muertos en el año*10000 }}{\mathrm{V}}$ 
$\mathrm{V}=\mathrm{TPD}(365)$

\section{Tabla 3. Índices de accidentalidad IA/K}

\begin{tabular}{cc}
\hline Año & IA/VEI \\
\hline 1 & 7,08 \\
\hline 2 & 5,52 \\
\hline
\end{tabular}

A medida que pasa el tiempo, se aumenta la severidad de un accidente por kilometraje de viaje $\mathrm{y}$ en intersecciones, a pesar de que se ha disminuido los accidentes en ellos, pero por factores de velocidad, imprudencia y falta de señalización, siguen siendo peligroso tomar intersecciones, según lo ilustrado en Tabla 3.

\subsection{Velocidad}

La velocidad y las distancias de seguridad entre el vehículo y los obstáculos desempeñan un papel fundamental en la Seguridad del Vehículo. Cuanto mayor sea la velocidad, mayor será la distancia de seguridad. A medida que aumenta la distancia de seguridad, disminuye la posibilidad de colisión entre el vehículo y el obstáculo (Huang et al., 2019), se puede observar en la Figura 3.

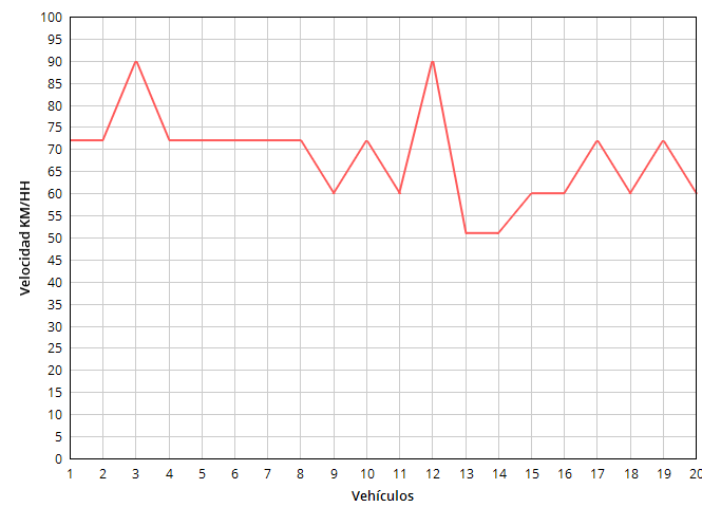

Fig 3. Velocidad de punto. Fuente: Elaboración propia.

Los vehículos analizados tiene un promedio de velocidad de $72 \mathrm{~km} / \mathrm{h}$, muy pocos vehículos exceden de este kilometraje, en cambio, prefieren ir con menos velocidad de punto.

\subsection{Volumen de tránsito}

El estudio de los volúmenes de tráfico en las intersecciones ocupadas, otorga una idea de la composición porcentual de los diferentes tipos de vehículos, la distribución direccional y el volumen de tráfico en hora punta (Kumar et al., 2017), por este motivo, se evidencia el volumen de tránsito en el año 1 y año 2 en la Tabla 4 y 5. $\underline{\text { Tabla 4. Volumen de tránsito - Año } 1}$

\begin{tabular}{|c|c|c|c|c|}
\hline Tiempo & Sentido & Volumen & Relación & $\begin{array}{l}\text { Producto } \\
\end{array}$ \\
\hline Día 1 & $\begin{array}{l}\text { Norte- } \\
\text { sur }\end{array}$ & 17648 & 2 & 35296 \\
\hline Día 1 & $\begin{array}{l}\text { Sur- } \\
\text { norte }\end{array}$ & 19479 & 2 & 38958 \\
\hline Día 2 & $\begin{array}{l}\text { Norte- } \\
\text { sur }\end{array}$ & 17429 & 2 & 34858 \\
\hline Día 2 & $\begin{array}{c}\text { Sur- } \\
\text { norte }\end{array}$ & 19840 & 2 & 39680 \\
\hline \multicolumn{4}{|c|}{ Transito promedio diario (TPD) } & 37198 \\
\hline
\end{tabular}

$\underline{\text { Tabla 5. Volumen de tránsito }-A \tilde{n} o 2}$

\begin{tabular}{|c|c|c|c|c|}
\hline Tiempo & Sentido & Volumen & Relación & Producto \\
\hline Día 1 & $\begin{array}{l}\text { Norte- } \\
\text { sur }\end{array}$ & 17628 & 2 & 35256 \\
\hline Día 1 & $\begin{array}{l}\text { Sur- } \\
\text { norte }\end{array}$ & 18736 & 2 & 374772 \\
\hline Día 2 & $\begin{array}{c}\text { Norte- } \\
\text { sur }\end{array}$ & 17417 & 2 & 34834 \\
\hline Día 2 & $\begin{array}{l}\text { Sur- } \\
\text { norte }\end{array}$ & 18881 & 2 & 37762 \\
\hline \multicolumn{4}{|c|}{ Transito promedio diario } & 36331 \\
\hline
\end{tabular}

\section{CONCLUSIONES}

El mayor riesgo de accidentes se dirige directamente a los conductores, quienes, al realizar acciones imprudentes durante la conducción, han recibido el mayor número de accidentes, por lo que se implementan alternativas para mitigar este riesgo, tales como la cultura u otras estrategias de sensibilización que podrían reducir el número de accidentes que se producen, la infraestructura se convierte entonces en una causa de menor impacto, sin embargo, se deben realizar las modificaciones pertinentes a la infraestructura de la carretera, con una programación adecuada y con el presupuesto correspondiente.

Con el tiempo, se ha demostrado que el porcentaje de los índices ha disminuido respecto al parque de vehículos, disminuyendo los accidentes y la morbilidad, pero aumentando el número de personas fallecidas en 0,76. Del mismo modo, los índices respecto a la población han disminuido en porcentaje en su tasa de siniestralidad y morbilidad, pero en mortalidad han aumentado un 0,51 respecto al año anterior.

Los indicadores de accidentes con respecto al kilometraje de viaje fueron muy similares entre los dos años, aumentaron en el segundo año 0.47, mientras que los vehículos que entraron a una intersección se redujeron en 2.06, aun así, la 
severidad de las intersecciones se redujo, debido a que no se han hecho cambios en la infraestructura. Muy pocos vehículos de más de $72 \mathrm{~km} / \mathrm{h}$, respetando la velocidad de un vehículo que pasa por un tramo, teniendo en cuenta que el volumen de tráfico diario entre los años 1 y 2 se redujo en 867 vehículos que pasan por este tramo, reduciendo el riesgo de accidentes entre peatones y otros vehículos.

\section{REFERENCIAS}

Liu, D. B., Wang, Y. X., \& Hua J. Y. (2018). Research on the Traffic Improvement Measures for Large Intersections. IOP Conference Series: Earth and Environmental Science, 189, 062075.

Bermeo, WL, Jr, AB de Souza, Fernández T, Honorio D, Nogueira dos Reis L, Barreto L. (2017). Control modo deslizante aplicado en la malla de corriente para una aplicación de una base-DSP para el control de posición de un motor de inducción de jaula de ardilla. Revista Tecnologías de Avanzada, ISSN: 1692-7257 Control modo deslizante aplicado en la malla de corriente para una aplicación de una base-DSP para el control de posición de un motor de inducción de jaula de ardilla. Revista Tecnologías de Avanzada, ISSN: 1692-7257

Szczuraszek, T., \& Klusek, R. (2019) Influence on the Type of Intersection on Road Traffic Safety in Poland. IOP Conference Series: Materials Science and Engineering, 471, 06202. 899X/471/6/062021

Roslan, R., Rusiman, M. S., Kamardan, M. G., Mohamad, N., Arobi, F. A., Che-Him, N., \& Khalid, K. (2018) Factors Affecting Road Traffic Accident in Batu Pahat, Johor, Malaysia. Journal of Physics: Conference Series, 995, 012033. https://doi.org/10.1088/17426596/995/1/012033

Dano, U. L. (2018) Improving Traffic Safety Towards Sustainable Built Environment in Dammam City, Saudi Arabia. IOP Conference Series: Earth and Environmental Science, $\quad 151, \quad 01203$. https://doi.org/10.1088/17551315/151/1/012031.

Farhan, M., Anas, M., \& Azeem, M. (2018) Rolling Barriers: Emerging Concept to Reduce Road Accidents: An Indian Perspective. IOP Conference Series: Materials Science and Engineering, 404,
012045. 899X/404/1/012045

Gaiginschi, L., Agape, I., \& Talif, S. (2017) Upon the reconstruction of accidents triggered by tire explosion. Analytical model and case study. IOP Conference Series: Materials Science and Engineering, 252, 012016. https://doi.org/10.1088/1757899X/252/1/012016

Suman, S., Shah, H., Susarla, V., \& Ravi, K. (2017) Numerical study for identification of influence of energy absorption and frontal crush for vehicle crashworthiness. IOP Conference Series: Materials Science and Engineering, 263, 062075. https://doi.org/10.1088/1757899X/263/6/062075

Pacheco, F., \& Mendoza, W. (2018) Análisis y Comportamiento del flujo vehicular en la carrera 10 del Municipio Los Patios, teniendo en Cuenta niveles de Accidentalidad y Parámetros de Tránsito. Trabajo de Pregrado, Universidad Francisco de Paula Santander, Colombia.

Huang, X., Shuai, C., \& Wu, X. (2019) Reactive navigation of autonomous vehicle. Journal of Physics: Conference Series, 1176, 052079. https://doi.org/10.1088/17426596/1176/5/05207900

Kumar, S. V., Gulati, H., \& Arora, S. (2017) Traffic Feasibility Study for a Grade Separator at a Busy Intersection in Vellore. IOP Conference Series: Materials Science and Engineering, 263, 032029. https://doi.org/10.1088/1757899X/263/3/032029 